\title{
THE STRUCTURE OF 0-E-UNITARY INVERSE SEMIGROUPS I: THE MONOID CASE
}

\author{
by MARK V. LAWSON
}

(Received 27th August 1997)

\begin{abstract}
This is the first of three papers in which we generalise the classical McAlister structure theory for $E$-unitary inverse semigroups to those 0 - $E$-unitary inverse semigroups which admit a 0 -restricted, idempotent pure prehomomorphism to a primitive inverse semigroup. In this paper, we concentrate on finding necessary and sufficient conditions for the existence of such prehomomorphisms in the case of 0 -E-unitary inverse monoids. A class of inverse monoids which satisfy our conditions automatically are those which are unambiguous except at zero, such as the polycyclic monoids.
\end{abstract}

1991 Mathematics subject classification: 20M18 (18B40).

\section{Introduction}

Zeros cause problems in semigroup theory. For this reason definitions in the field often come in pairs: a "definition" for semigroups without zero and a "0-definition" for those semigroups with zero. Here "without zero" is shorthand for "without a distinguished zero". Thus there are "simple semigroups" and "0-simple semigroups" and "bisimple semigroups" and "0-bisimple semigroups".

The class of " $E$-unitary inverse semigroups" was without such a companion until in 1987 Mária Szendrei [31] introduced the class of " $E^{*}$-unitary inverse semigroups", which are precisely the " 0 - $E$-unitary inverse semigroups", the term we shall use in this paper. An inverse semigroup with zero is 0-E-unitary when any element above a nonzero idempotent in the natural partial order is itself an idempotent.

The late recognition of the class of $0-E$-unitary inverse semigroups is probably due to the fact that $E$-unitary inverse semigroups have long been viewed as building blocks for the whole class of inverse semigroups, and so there seemed no reason to deal with a more general class of semigroups. In fact, $E$-unitary inverse semigroups are primarily interesting because so many naturally occurring inverse semigroups are $E$-unitary. This is also true of $0-E$-unitary semigroups.

Elementary examples of 0 - $E$-unitary inverse semigroups are $E$-unitary inverse semigroups with a zero adjoined and all primitive inverse semigroups. More interestingly, all polycyclic monoids on at least two generators are 0 - $E$-unitary, a property first noted by Meakin and Sapir [26]; the polycyclic monoid on one generator is the bicyclic monoid which is $E$-unitary. Recently, and remarkably, the class of $0-E$ - 
unitary inverse semigroups has been enormously expanded with the advent of "tiling semigroups". Johannes Kellendonk $[10,11,12]$ has shown how to construct a $0-E$ unitary inverse semigroup from every tiling in $\mathbb{R}^{n}$. Such semigroups are subsequently used to construct invariants of the tiling important in mathematical physics.

These examples suggest that it is time to study $0-E$-unitary inverse semigroups, not as potential building blocks for other semigroups, but as an important and interesting class of inverse semigroups in their own right.

There has already been some work in this direction. Gomes and Howie [7] studied those $0-E$-unitary semigroups which are categorical at zero. This assumption has the effect of constraining the waywardness of the zero, and so enables the classical theory of $E$-unitary inverse semigroups to be generalised relatively easily. In particular, $0-E$ unitary inverse semigroups which are categorical at zero admit a 0-restricted idempotent pure homomorphism to a primitive inverse semigroup. However, it is a very strong assumption. No polycyclic monoid on two or more generators satisfies it nor do Kellendonk's tiling semigroups. Furthermore, the polycyclic monoid on two or more generators is congruence-free. This strongly suggests that homomorphisms may be the wrong morphisms to use in the general case.

We need a different approach which will contain as many naturally occurring examples of $0-E$-unitary semigroups as possible. To discover how to do this, let me briefly recall the two important elements of the classical theory of $E$-unitary inverse semigroups [29]:

1. An inverse semigroup $S$ is $E$-unitary if, and only if, there exists a surjective, idempotent pure homomorphism to a group.

2. There is a structure theory for idempotent pure homomorphisms which enables $S$ to be described in terms of "McAlister triples". This is McAlister's P-theorem.

Any generalisation of the classical theory will have to generalise each of these two elements. The key to my generalisation is an analysis of the first element.

Our comments earlier suggest that we should abandon homomorphisms and look instead for a wider class of functions which are nevertheless close enough to homomorphisms to be tractable. There were two clues to the nature of these functions. Firstly, although the polycyclic monoid on two generators does not admit any (group) congruences it is easy to find prehomomorphisms to groups, indeed there is a 0restricted, idempotent pure prehomomorphism to the free group on two generators. Secondly, in Kellendonk's theory of tilings it is the 0-restricted prehomomorphisms which are the main morphisms considered. Before continuing let me remind the reader of the salient definitions.

A function $\theta: S \rightarrow T$ is said to be a prehomomorphism if $\theta(x y) \leq \theta(x) \theta(y)$ for all $x, y \in S$. It is said to be 0 -restricted if $\theta^{-1}(0)=\{0\}$, and it is said to be idempotent pure if $\theta(x)$ an idempotent implies $x$ is an idempotent.

Terminology. I shall often refer to 0-restricted, idempotent pure prehomomorphisms as "suitable". 
Thus the central task of the first two papers in our trilogy can now be stated: we shall investigate the existence and behaviour of suitable prehomomorphisms from $0-E$ unitary inverse semigroups to primitive inverse semigroups. In this paper we shall deal solely with the simpler case of $0-E$-unitary inverse monoids and tackle the more complicated general case in the next paper.

What then of the second element? Here we were lucky. I have already developed a theory of idempotent pure prehomomorphisms based on Ehresmann's maximum enlargement theorem $[13,14,15,16]$. For this reason, I shall deal with this theory in the third paper in the sequence.

In the recent preprint [3], the authors have also recognised the need to replace homomorphisms by more general maps. They use what they term "semihomomorphisms". In fact, these are prehomomorphisms. However, their paper assumes that a $0-E$-unitary inverse semigroup is already equipped with a suitable prehomomorphism to a 0-group. Thus their paper is essentially concerned with a special case of the second part of the program outlined above.

The paper is divided into two sections. In Section 2, the essential ideas of my approach are introduced without the technical apparatus by concentrating on 0 bisimple inverse monoids. I show that a 0 - $E$-unitary 0 -bisimple inverse monoid admits a suitable prehomomorphism if, and only if, the $\mathcal{R}$-class containing the identity can be embedded in a group. These results are then systematically generalised to arbitrary 0 E-unitary inverse monoids in Section 3.

I would like to thank Johannes Kellendonk of the T.U. Berlin for numerous conversations on the structure of 0-E-unitary inverse semigroups, and Vicky Gould and John Fountain of the University of York, England for some helpful remarks.

\section{A special case: 0 -bisimple inverse monoids}

The aim of this section is to find necessary and sufficient conditions for the existence of a suitable prehomomorphism from a 0 - $E$-unitary 0 -bisimple inverse monoid to a primitive inverse semigroup (Theorem 8 ). The theory is then illustrated by applying it to the polycyclic monoids.

We begin by recalling the standard properties of prehomomorphisms we shall need. Recall that the restricted product (also called the "trace product") is a partial binary operation defined in any inverse semigroup as follows: $x \cdot y$ exists and is equal to $x y$ precisely when $x^{-1} x=y y^{-1}$. An important property of restricted products which is readily checked is that

$$
x \cdot y \mathcal{L} y \text { and } x \cdot y \mathcal{R} x
$$

The proofs of the following may be found in [24] and [25].

Proposition 1. If $\theta$ is a prehomomorphism from $S$ to $T$ then the following hold.

(i) $\theta\left(s^{-1}\right)=\theta(s)^{-1}$ for all $s \in S$. 
(ii) $\theta$ preserves the restricted product.

(iii) If $x^{-1} x \leq y y^{-1}$ or $y y^{-1} \leq x^{-1} x$ then $\theta(x y)=\theta(x) \theta(y)$.

(iv) $\theta$ preserves the natural partial order.

(v) $\theta$ maps $\mathcal{L}$-related (resp. $\mathcal{R}$-related) elements to $\mathcal{L}$-related (resp. $\mathcal{R}$-related) elements.

(vi) $\theta$ is idempotent pure if, and only if, all restrictions of $\theta$ to $\mathcal{L}$-classes (resp. $\mathcal{R}$ classes) are injective.

Property (iii) above plays a crucial role in our work.

The exact relationship between prehomomorphisms and homomorphisms is described by the following result. It is a consequence of Proposition 1 and the fact that every product $x y$ can be written in the form $x y=(x e) \cdot(e y)$ where $e=x^{-1} x y y^{-1}$.

Proposition 2. A function $\theta: S \rightarrow T$ between inverse semigroups is a prehomomorphism if, and only if, it preserves the restricted product and the natural partial order. A prehomomorphism is a homomorphism precisely when $\theta(e f)=\theta(e) \theta(f)$ for all idempotents $e, f \in S$.

The classical structure theory of 0-bisimple inverse monoids due to Clifford [4], Reilly [30] and McAlister [23] tells us that the properties of a 0-bisimple inverse monoid are completely determined by the properties of the $\mathcal{R}$-class containing the identity. Theorem 3 below summarises some aspects of this relationship. The proofs are included because they are the basis of my generalisation to arbitrary 0 - $E$-unitary inverse monoids in Section 3.

Terminology. In this section I shall use the term "CRM-monoid" (named after Clifford, Reilly and McAlister) to mean a right cancellative monoid with the property that any two principal left ideals are either disjoint or intersect in a principal left ideal.

Theorem 3. Let $S$ be a 0-bisimple inverse monoid with identity 1 and put $C=R_{1}$.

(i) C is a CRM-monoid.

(ii) If $a, b \in C$ then $a \mathcal{L}(S) b$ if, and only if, there is an invertible element $u \in H_{1}$ such that $a=u b$.

(iii) $S \backslash\{0\}=C^{-1} C$.

(iv) Let $a, b, c, d \in C$. Then $b^{-1} a=d^{-1} c$ if, and only if, there is an invertible element $u \in H_{1}$ such that $a=u c$ and $b=u d$.

(v) $b^{-1} a$ is an idempotent if, and only if, $a=b$.

(vi) Let $x=b^{-1} a$ and $y=d^{-1} c$. Then $x \leq y$ if, and only if, $b=p d$ and $a=p c$ for some $p \in C$. 
Proof. (i) First note that $C$ contains the idempotent 1 and so is non-empty. Let $a, b \in C$. In particular $b \mathcal{R} 1$. But $\mathcal{R}$ is a left congruence so that $a b \mathcal{R} a 1=a$ and $a \mathcal{R} 1$. Thus $a b \mathcal{R} 1$. Hence $C$ is closed under multiplication and so it is a monoid with identity 1. Now suppose that $a c=b c$ where $a, b, c \in C$. Then $a c c^{-1}=b c c^{-1}$. But $c c^{-1}=1$, and so $a=b$, hence $C$ is a right cancellative monoid.

Before proving the assertion on the behaviour of the principal left ideals under intersection, we first prove that $C x=S x \cap C$ for all $x \in C$. Let $y \in S x \cap C$. Then $y \mathcal{R} 1$ and $y=s x$ for some $s \in S$. Thus $y=\left(y x^{-1}\right) x$. But

$$
y x^{-1}\left(y x^{-1}\right)^{-1}=y x^{-1} x y^{-1}=s x x^{-1} x x^{-1} s^{-1}=s x x^{-1} s^{-1}=y y^{-1}=1
$$

and so $y x^{-1} \in C$. Hence $y \in C x$.

Conversely, let $y \in C x$. Then $y=a x$ where $a \in C$. Clearly $y \in S x$. Also

$$
y y^{-1}=(a x)(a x)^{-1}=a x x^{-1} a^{-1}=a a^{-1}=1
$$

so that $y \in C$. Thus $y \in S x \cap C$.

It remains to prove that if $C a \cap C b$ is non-empty for any $a, b \in C$ then there exists $c \in C$ such that $C a \cap C b=C c$. It is easy to check that $S x \cap S y=S x^{-1} x y^{-1} y$ for all $x, y \in S$. Thus

$$
C x \cap C y=S\left(x^{-1} x y^{-1} y\right) \cap C .
$$

The righthand-side is empty precisely when $x^{-1} x y^{-1} y=0$; to see this, suppose first that $x^{-1} x y^{-1} y$ is non-zero. Then there exists $z \in L_{x^{-1} x y^{-1} y} \cap C$, since $S$ is 0 -bisimple, in which case

$$
C x \cap C y=S z \cap C=C z,
$$

and is non-empty. Conversely, suppose that $w \in S x^{-1} x y^{-1} y \cap C$. Then $w\left(x^{-1} x y^{-1} y\right)=w$ and $w \mathcal{R} 1$. Since 1 is non-zero, $w$ must be non-zero. But then $x^{-1} x y^{-1} y$ must be non-zero.

(ii) If $a \mathcal{L} b$ then $a^{-1} a=b^{-1} b$. Thus $a=a a^{-1} a=a\left(b^{-1} b\right)=\left(a b^{-1}\right) b$. But $a b^{-1}$ is a restricted product and so it is $\mathcal{H}$-related to 1 . The converse is clear.

(iii) Let $x \in S \backslash\{0\}$. Then $x \mathcal{D} 1$ and so $x \mathcal{L} a \mathcal{R} 1$ for some $a \in S \backslash\{0\}$. Thus $x^{-1} x=a^{-1} a$ and $a a^{-1}=1$. In particular, $a \in C$. Thus

$$
x=x x^{-1} x=x\left(a^{-1} a\right)=\left(x a^{-1}\right) a=\left(a x^{-1}\right)^{-1} a .
$$

But

$$
\left(a x^{-1}\right)\left(a x^{-1}\right)^{-1}=a x^{-1} x a^{-1}=a\left(a^{-1} a\right) a^{-1}=1 .
$$

If we put $b=a x^{-1}$ then we have shown that $a, b \in C$ and $x=b^{-1} a$. 
(iv) Suppose that $b^{-1} a=d^{-1} c$. Then $b \mathcal{L} d$, and so there is an invertible element $u \in H_{1}$ such that $b=u d$. Now

$$
a=a a^{-1} a=b b^{-1} a=b d^{-1} c=u c .
$$

The converse is clear.

(v) Suppose that $b^{-1} a$ is an idempotent. Then $b a^{-1} b a^{-1}=b a^{-1}$. It is straightforward to show that $a=a b^{-1} a$ and so $a b^{-1} b=b$. Thus $b \leq a$. But $a \mathcal{R} b$ and so $a=b$.

(vi) Suppose that $x \leq y$. Then $x=x x^{-1} y$. From which we obtain $a=\left(b d^{-1}\right) c$. Put $p=b d^{-1}$. Then $a a^{-1}=p c(p c)^{-1}=p c c^{-1} p^{-1}=p p^{-1}$. Thus $p \in C$. Also $b^{-1} b \leq d^{-1} d$ and so $p d=b d^{-1} d=b$. The converse is clear.

The following result, which may be deduced from [23] and [30], contains the essentials of the classical theory of 0 -bisimple inverse monoids.

Theorem 4. For every CRM-monoid $C$ there is a 0-bisimple inverse monoid $S$ such that $C=R_{1}$, which is unique up to isomorphism.

The $R_{1}$-class of a 0 -bisimple inverse monoid determines the structure of the monoid as a whole. In particular, 0 - $E$-unitary bisimple inverse monoids have a characterisation in these terms which is important for our work.

Theorem 5. Let $S$ be a 0-bisimple inverse monoid. Put $C=R_{1}$. Then $S$ is 0 -E-unitary if, and only if, $C$ is a cancellative monoid.

Proof. Let $S$ be 0 -E-unitary and let $c a=c b$ where $a, b, c \in C$. Then $c^{-1} c a=c^{-1} c b$ and so

$$
a^{-1} c^{-1} c a=a^{-1} c^{-1} c b a^{-1} \leq a^{-1} b .
$$

Suppose that $a^{-1} c^{-1} c a=0$. Then $0=a a^{-1} c^{-1} c a a^{-1}=c^{-1} c$, which implies that $c=0$, a contradiction. Thus $a^{-1} c^{-1} c a$ is a non-zero idempotent, and so $a^{-1} b$ is an idempotent $f$, say, since $S$ is $0-E$-unitary. But then $a f=a a^{-1} b=b$ and so $b \leq a$. By assumption $a \mathcal{R} b$, and so $a=b$.

Conversely, suppose that $C$ is cancellative. Let $e \leq x$ where $e$ is a non-zero idempotent. Then by Theorem 3, we can write $e=a^{-1} a$ and $x=b^{-1} c$ where $a, b, c \in C$. Also by Theorem 3, there exists $p \in C$ such that $(a, a)=p(b, c)$. Thus $a=p b=p c$. By assumption $C$ is left cancellative and so $b=c$. Thus $x=b^{-1} b$ is an idempotent.

From the results above, we deduce that the theory of 0 -E-unitary 0 -bisimple inverse monoids is inextricably bound up with the theory of cancellative CRM-monoids.

In the next two propositions, we shall find necessary and sufficient conditions respectively for the existence of a 0 -restricted, idempotent pure prehomomorphism from a 0 -E-unitary, 0 -bisimple inverse monoid to a primitive inverse semigroup. 
Proposition 6. Let $\theta: S \rightarrow T$ be a suitable prehomomorphism from a 0 -E-unitary 0 bisimple inverse monoid $S$ to a primitive inverse semigroup $T$ and put $C=R_{1}$.

(i) The images of every non-zero element of $S$ lie within a single (group) $\mathcal{H}$-class $G$ of $T$.

(ii) The cancellative monoid $C$ is embedded in the group $G$.

Proof. (i) Let $e$ be any non-zero idempotent. Then $e \leq 1$. Prehomomorphisms preserve the natural partial order by Proposition 1 so that $\theta(e) \leq \theta(1)$. Neither $\theta(e)$ nor $\theta(1)$ is zero since $\theta$ is 0 -restricted. But $S$ is a primitive inverse semigroup, consequently $\theta(e)=\theta(1)$. Hence all non-zero idempotents of $S$ have the same image in $T$. If $x$ is a non-zero element of $S$ then neither $x^{-1} x$ nor $x x^{-1}$ is zero and so $\theta\left(x^{-1} x\right)=$ $\theta(1)=\theta\left(x x^{-1}\right)$. However, prehomomorphisms preserve the $\mathcal{L}$ - and $\mathcal{R}$-relations by Proposition 1, and so $\theta(x) \mathcal{H} \theta(1)$.

(ii) Putting $G=H_{0(1)}$, the prehomomorphism restricts to a function from $C$ to $G$. It is injective because $\theta$ is idempotent pure and so injective when restricted to $\mathcal{R}$-classes of $S$ by Theorem 1. If $a, b \in C$ then we always have $a^{-1} a \leq b b^{-1}=1$, and so $\theta$ is a homomorphism when restricted to $C$ by Theorem 1. Thus $C$ is homomorphically embedded in $G$.

A necessary condition for the existence of a suitable prehomomorphism is therefore that the $\mathcal{R}$-class containing the identity be embeddable in a group. We shall now show that this is also sufficient.

Proposition 7. Let $S$ be a 0 -E-unitary inverse monoid with the property that $C=R_{1}$ can be embedded in a group. Then there exists a 0-restricted idempotent pure prehomomorphism from $S$ to a primitive inverse semigroup. Moreover, the primitive inverse semigroup can be chosen to be a group with zero adjoined (a 0-group).

Proof. Let $\phi$ be an embedding of $C$ into a group $G$. We define a function $\theta: S \rightarrow G^{0}$ as follows: $\theta(0)=0$ and if $x \in S$ is non-zero and $x=b^{-1} a$ where $a, b \in C$ then define $\theta(x)=\phi(b)^{-1} \phi(a)$. To prove that $\theta$ is well-defined, suppose that

$$
x=b^{-1} a=d^{-1} c .
$$

By Theorem 3 there is an invertible element $u \in C$ such that $b=u d$ and $a=u c$. Thus

$$
\phi(b)^{-1} \phi(a)=\phi(u d)^{-1} \phi(u c)=(\phi(u) \phi(d))^{-1} \phi(u) \phi(c)=\phi(d)^{-1} \phi(c),
$$

and so $\theta(x)$ is defined independently of its representation.

The function $\theta$ is 0 -restricted by virtue of its definition. Observe that all non-zero idempotents of $S$ are mapped to the identity of $G^{0}$. Now suppose that $\theta(x)=1$. Let $x=b^{-1} a$. Then 


$$
\theta(x)=\phi(b)^{-1} \phi(a)=1 \text {. }
$$

Thus $\phi(a)=\phi(b)$. But $\phi$ is injective and so $a=b$ and $x$ is an idempotent.

It remains to prove that $\theta$ is a prehomomorphism. We shall do this by showing that $\theta$ preserves the natural partial order and the restricted product. Let $x, y \in S$ be nonzero elements, where $x=b^{-1} a$ and $y=d^{-1} c$. Suppose that $x \leq y$. Then $a=p c$ and $b=p d$ for some $p \in C$ by Theorem 3. Thus

$$
\theta(x)=(\phi(p d))^{-1} \phi(p c)=\phi(d)^{-1} \phi(p)^{-1} \phi(p) \phi(c)=\phi(d)^{-1} \phi(c)=\theta(y) .
$$

It is now clear that $\theta$ preserves the natural partial order.

Suppose that $x y$ is a restricted product where $x=b^{-1} a$ and $y=d^{-1} c$. Then $a^{-1} a=d^{-1} d$ so that there exists an invertible element $u \in C$ such that $a=u d$. Thus

$$
x y=b^{-1} a d^{-1} c=b^{-1} u d d^{-1} c=b^{-1}(u c) .
$$

Thus

$$
\theta(x y)=\phi(b)^{-1} \phi(u c)=\phi(b)^{-1} \phi(u) \phi(c)
$$

and

$$
\theta(x) \theta(y)=\phi(b)^{-1} \phi(a) \phi(d)^{-1} \phi(c)=\phi(b)^{-1} \phi(u) \phi(c) .
$$

Hence $\theta$ preserves the restricted product.

We may summarise the results of Propositions 6 and 7 as follows.

Theorem 8. Let $S$ be a 0-E-unitary 0-bisimple inverse monoid. Then $S$ admits a 0 restricted, idempotent pure prehomomorphism to a primitive inverse semigroup if, and only if, $R_{1}$ can be embedded in a group.

If a 0 - $E$-unitary 0 -bisimple inverse monoid admits a suitable prehomomorphism then we would like to find a canonical such prehomomorphism. To do this we need the concept of the "fundamental group" of a monoid [22].

Theorem 9. For every monoid $M$ there exists a group $U(M)$ and a monoid homomorphism $\eta: M \rightarrow U(M)$ such that $\eta(M)$ generates $U(M)$ and for every homomorphism $\theta: M \rightarrow G$ to a group $G$ such that $\theta(M)$ generates $G$ there exists a unique homomorphism $\theta^{*}: U(M) \rightarrow G$ such that $\theta^{*} \eta=\theta$.

The group $U(M)$ is called the fundamental group of the monoid $M$. The key result from our point of view is the following. Whether a monoid $M$ can be embedded in a group or not is controlled by the homomorphism $\eta: M \rightarrow U(M)$. The proof of the following is straightforward or may be found in [5]. 
Proposition 10. A monoid $M$ can be embedded in a group if, and only if, it is embedded in $U(M)$ by $\eta$.

Let $S$ be a 0 -bisimple 0 - $E$-unitary monoid with the property that $R_{1}$ is embedded in $G=U\left(R_{1}\right)$ by $\eta$. Let $\beta: S \rightarrow G^{0}$ be the suitable prehomomorphism constructed from $\eta$ by means of Proposition 7 . It is characterised by the following property.

Theorem 11. Let $\theta: S \rightarrow T$ be any suitable prehomomorphism to a primitive inverse semigroup $T$ such that the image of $\theta$ generates $T$. Then there is a unique 0 -restricted prehomomorphism $\theta^{*}: G^{0} \rightarrow T$ such that $\theta^{*} \beta=\theta$.

Proof. By Proposition 6, the primitive inverse semigroup $T$ must be a group with zero, $H^{0}$, say. Also by Proposition $6, \theta$ must restrict to an embedding of $R_{1}$ in $H$. Because every non-zero element of $S$ can be written as a product of the form $b^{-1} a$ where $a, b \in R_{1}$ by Theorem 3 it follows that the image of $\left(\theta \mid R_{1}\right)$ generates $H$. Thus by Theorem 9, there exists a unique group homomorphism $\theta^{*}: G \rightarrow H$ such that $\theta^{*} \eta=$ $\left(\theta \mid R_{1}\right)$. We may extend $\theta^{*}$ by defining $\theta^{*}(0)=0$. In this way, we obtain a 0 -restricted (pre)homomorphism from $G^{0}$ to $H^{0}$.

We now check that $\theta^{*} \beta=\theta$. Let $s \in S$ be a non-zero element. Then $s=a^{-1} b$ where $a, b \in R_{1}$. By Proposition 7, we have that

$$
\theta^{*}(\beta(s))=\theta^{*}\left(\eta(a)^{-1} \eta(b)\right)
$$

which is equal to $\theta(a)^{-1} \theta(b)$. But $a^{-1} b$ is a restricted product and $\theta$ is a prehomomorphism and so $\theta\left(a^{-1} b\right)=\theta(a)^{-1} \theta(b)$. Hence $\theta^{*}(\beta(s))=\theta(s)$, as required. It is clear that $\theta^{*}$ is unique with the stated properties.

The above theorem reassures as that if a 0 -bisimple inverse monoid admits a suitable prehomomorphism to a primitive inverse semigroup then it possesses a universal such prehomomorphism. This universal prehomomorphism would be a natural candidate to replace the minimum group congruence in the case of inverse semigroups without zero.

Necessary and sufficient conditions for embedding monoids in groups were discovered by Mal'cev [6]. We shall not pursue this theory here. Instead, we shall examine two examples of sufficient conditions. A proof of the first may be found in [6].

Theorem 12. Let $C$ be a cancellative monoid satisfying Ore's condition: for all $x, y \in C$ there exist elements $a, b \in C$ such that $a x=b y$. Then $C$ can be embedded in $a$ group; in particular, $U(C)=\eta(C)^{-1} \eta(C)$, and so is a group of fractions of $C$.

The above result enables us to understand why the existence of suitable prehomomorphisms for $E$-unitary bisimple inverse monoids is automatic. 
Theorem 13. Let $S$ be an E-unitary, bisimple inverse monoid. Then the cancellative monoid $C=R_{1}$ satisfies Ore's condition, and $S$ admits an idempotent pure homomorphism to a group. In particular, $S / \sigma$ is isomorphic to the fundamental group of $R_{1}$.

Proof. Since $S$ has no zero the intersection of any two principal left ideals of $C$ is again a principal left ideal. Since $S$ is $E$-unitary $C$ is cancellative. Thus $C$ certainly satisfies Ore's conditions, and so possesses a group of fractions. The standard theory of inverse semigroups informs us that the minimum group congruence $\sigma$ is such that $\sigma^{\natural}: S \rightarrow S / \sigma$ is a surjective idempotent pure homomorphism. Let $\eta: C \rightarrow G$ be the embedding of $C$ in its group of fractions. The corresponding prehomomorphism $\beta: S \rightarrow G$ is actually a homomorphism because there is no zero, and is surjective because every element of $G$ is of the form $\eta(a)^{-1} \eta(b)$ for some $a, b \in C$. Thus $\sigma \subseteq \operatorname{ker}(\theta)$ since $\sigma$ is the minimum group congruence. However, by the universal property of $\beta$ guaranteed by Theorem 11 , there is a homomorphism $\left(\sigma^{\natural}\right)^{*}: G \rightarrow S / \sigma$ such that $\left(\sigma^{\natural}\right)^{*} \beta=\sigma^{*}$, from which we deduce that $\operatorname{ker}(\beta) \subseteq \sigma$. Thus $G$ and $S / \sigma$ are isomorphic.

Von Karger [9] discovered another class of cancellative monoids which can be embedded in groups.

Theorem 14. Let $C$ be a cancellative monoid satisfying the mediation condition: if two principal left ideals intersect non-emptily then one is contained in the other. Then $C$ can be embedded in a group.

The above theorem and the simple theory I have developed so far can be used to shed some light on the behaviour of polycyclic monoids [28]. To describe these results, we need to recall some results on free monoids.

Let $\Sigma$ be any non-empty set called an alphabet. The set $\Sigma^{*}$ of all finite sequences of elements of $\Sigma$ is the free monoid on $\Sigma$ under the operation of concatenation and with the identity being the empty sequence, denoted by 1 . Elements of $\Sigma^{*}$ are called strings over $\Sigma$. A typical non-empty element of $\Sigma^{*}$ is thus of the form $\left(x_{1} \ldots x_{n}\right)$ where $x_{i} \in \Sigma$. The length of this string is $n$. Free monoids are cancellative. The free monoid on one generator is isomorphic to the natural numbers under addition; the isomorphism maps each string to its length. Let $x$ be a string in $\Sigma^{*}$. If $x=y z$ for some string $y$, then we say that $z$ is a suffix of $x$.

Define the following binary operation on $\Sigma^{*}$. Let $x, y \in \Sigma^{*}$. Then

$$
(y \backslash x)= \begin{cases}u & \text { if } y=u x \\ 1 & \text { otherwise }\end{cases}
$$

In the case of the free monoid on one generator, this operation reduces to the "monus" operation $a-b$ which is zero if $b>a$ and $a-b$ otherwise.

The proof of the following is straightforward.

Lemma 15. Let $x$ and $y$ be strings in $\Sigma$. Then $\Sigma^{*} u \cap \Sigma^{*} v$ is non-empty precisely when 
either $u$ is a suffix of $v$ or $v$ is a suffix of $u$. If this is the case, then $(v \backslash u) u=(u \backslash v) v$, and

$$
\Sigma^{*} u \cap \Sigma^{*} v=\Sigma^{*}(v \backslash u) u=\Sigma^{*}(u \backslash v) v .
$$

The polycyclic monoids may now be described. Let $n \geq 2$ and let $\Sigma$ be an alphabet with $n$ elements. The polycyclic monoid $P_{n}$ on $n$ generators is isomorphic to the set $\Sigma^{*} \times \Sigma^{*} \cup\{0\}$ with the following multiplication:

$$
(x, y)(u, v)= \begin{cases}((u \backslash y) x,(y \backslash u) v) & \text { if } \Sigma^{*} y \cap \Sigma^{*} u \neq \emptyset \\ 0 & \text { else }\end{cases}
$$

The element 0 is defined to act as a zero. For $n=1$, let $\Sigma$ be an alphabet with one element. The monoid $P_{1}$, the bicyclic monoid, is $\Sigma^{*} \times \Sigma^{*}$ with the multiplication above, except that the zero is omitted. In view of the isomorphism between the free monoid on a one-letter alphabet and the natural numbers, the bicyclic monoid can be regarded as the set $\mathbb{N} \times \mathbb{N}$ with the product:

$$
(a, b)(c, d)=((c-b)+a,(b-c)+d) .
$$

We can now analyse the polycyclic monoids. Since the non-zero idempotents are the elements of the form $(u, u)$ and the natural partial order is given by

$$
(u, v) \leq(x, y) \Leftrightarrow(u, v)=(p x, p y)
$$

for some $p \in \Sigma^{*}$ it is evident that $P_{1}$ is $E$-unitary and $P_{n}$ is 0 - $E$-unitary for $n \geq 2$. Consider the bicyclic monoid $P_{1}$. The CRM-monoid here is $(\mathbb{N},+)$. It is clear that Ore's condition holds and the group of fractions of $\mathbb{N}$ is $\mathbb{Z}$. Thus, as we know, there is an idempotent pure homomorphism from $P_{1}$ to $\mathbb{Z}$.

We shall now treat uniformly all polycyclic monoids $P_{n}$ where $n \geq 2$. The CRMmonoid here is the free monoid on $n$ generators. This does not satisfy Ore's condition, but it does satisfy the mediation condition of Theorem 14. In fact, we know already that the free monoid on $n$ generators can be embedded into the free group $F G_{n}$ on $n$ generators. Thus according to the theory developed in Proposition 7, we can define a suitable prehomomorphism $\theta: P_{n} \rightarrow F G_{n}^{0}$ by $\theta(0)=0$ and $\theta(u, v)=\operatorname{red}\left(u^{-1} v\right)$ where $\operatorname{red}(x)$ is the reduced string corresponding to $x$. Observe that the image of $\theta$ generates $F G_{n}$ because it contains all elements of the form $\theta(1, x)=x$ where $x \in \Sigma$. This map also works in the case of $P_{1}$ because then $\theta(m, n)=n-m \in \mathbb{Z}$. It is easy to check that

$$
(m, n) \sigma(p, q) \Leftrightarrow n-m=q-p .
$$

The results of this section have led us to consider those 0-E-unitary 0-bisimple inverse monoids $S$ which admit 0-restricted idempotent pure prehomomorphisms $\theta: S \rightarrow G^{0}$ to 0 -groups such that the image of $\theta$ generates $G^{0}$. 


\section{Arbitrary inverse monoids}

In this section, we shall obtain necessary and sufficient conditions for a $0-E$-unitary inverse monoid to admit a suitable prehomomorphism to a primitive inverse semigroup (Theorem 11).

0-bisimple inverse monoids can be completely described in terms of the right cancellative monoid $R_{1}$ which is a CRM-monoid. The analogue of such monoids for arbitrary inverse monoids we call "CRM-categories". Before defining these, we begin by recalling the definition of a "category" regarded as a generalisation of a monoid.

Let $C$ be a set equipped with a partial binary operation which we shall denote by . or by concatenation. If $x, y \in C$ and the product $x \cdot y$ is defined we write $\exists x \cdot y$. An element $e \in C$ is called an identity if $\exists e \cdot x$ implies $e \cdot x=x$ and $\exists x \cdot e$ implies $x \cdot e=x$. The set of identities of $C$ is denoted $C_{0}$. The pair $(C, \cdot)$ is said to be a category if the following axioms hold:

(C1) $x \cdot(y \cdot z)$ exists if, and only if, $(x \cdot y) \cdot z$ exists, in which case they are equal.

(C2) $x \cdot(y \cdot z)$ exists if, and only if, $x \cdot y$ and $y \cdot z$ exist.

(C3) For each $x \in C$ there exist identities $e$ and $f$ such that $\exists x \cdot e$ and $\exists f \cdot x$.

From (C3), it can easily be deduced that the identities $e$ and $f$ are uniquely determined by $x$. We write $e=\mathrm{d}(x)$ and $f=\mathrm{r}(x)$. Observe that $\exists x \cdot y$ if, and only if, $\mathbf{d}(x)=\mathbf{r}(y)$. If $C$ is a category and $e$ and $f$ identities in $C$ then we put

$$
\operatorname{hom}(e, f)=\{x \in C: \mathbf{d}(x)=e \text { and } \mathbf{r}(x)=f\},
$$

the set of all homomorphisms from $e$ to $f$ or hom-set. We also put end $(e)=\operatorname{hom}(e, e)$, the endomorphism monoid at e. A weak initial identity in a category is an identity 1 such that $\operatorname{hom}(1, e)$ is non-empty for all identities $e$.

A pair of elements $s$ and $t$ in a category with a common domain will be said to be completable to a commutative square if there are elements $u$ and $v$ such that $u s=v t$.

An element $x \in C$ is said to be invertible or an isomorphism if there exists $x^{-1} \in G$ such that $x^{-1} x=\mathrm{d}(x)$ and $x x^{-1}=\mathbf{r}(x)$. A groupoid is a category in which every element is invertible. A groupoid is said to be connected if for any two identities $e$ and $f$ the hom-set $\operatorname{hom}(e, f)$ is non-empty.

A category is said to be right cancellative if $x \cdot a=y \cdot a$ implies $x=y$, and left cancellative if $a \cdot x=a \cdot y$ implies $x=y$. A cancellative category is one which is both left and right cancellative.

We begin with a simple lemma. The proof is straightforward.

Lemma 1. Let $S$ be an inverse semigroup with zero.

(i) $S s \cap S t=S s^{-1} s t^{-1} t$ for all $s, t \in S$.

(ii) $S s^{-1} s t^{-1} t=\{0\}$ if, and only if, $s t^{-1}=0$.

(iii) For all $s, t \in S$ either $S s \cap S t=\{0\}$ or there exists $w \in S$ such that $S s \cap S t=S w$. 
A category $C$ is said to be a $C R M$-category if it satisfies the following conditions.

(CRM1) $C$ is right cancellative.

(CRM2) $C$ has a weak initial identity.

(CRM3) Any pair of elements $s, t \in C$ with a common domain which can be completed to a commutative square have a pushout.

We shall show that every inverse monoid with zero $S$ gives rise to a CRM-category $C_{1}(S)$. Our construction of the category $C_{1}(S)$ is a modification of one due to Leech [20]. He constructs a category using all elements of an inverse semigroup. It is important in our work that the zero be omitted. We then have to check that this is possible and determine the properties of the resulting category.

Let $S$ be an inverse semigroup with zero. Put

$$
C_{1}(S)=\left\{(s, e) \in S \times E(S): s^{-1} s \leq e \text { and } s, e \neq 0\right\} .
$$

Define

$$
\mathrm{d}(s, e)=(e, e) \text { and } \mathrm{r}(s, e)=\left(s s^{-1}, s s^{-1}\right)
$$

and a partial product on $C_{1}(S)$ by

$$
(s, e) \cdot(t, f)=\left\{\begin{array}{l}
(s t, f) \text { if } e=t t^{-1} \\
\text { undefined else. }
\end{array}\right.
$$

Theorem 2. (i) $\left(C_{1}(S), \cdot\right)$ is a right cancellative category.

(ii) The isomorphisms in $C_{1}(S)$ are the elements of the form $\left(s, s^{-1} s\right)$.

(iii) Any two elements of $C_{1}(S)$ with a common domain which can be completed to a commutative square have a pushout.

Proof. (i) We first have to show that if the partial product exists then the result also belongs to $C_{1}(S)$. Suppose that $\exists(s, e) \cdot(t, f)$. Then $(s, e) \cdot(t, f)=(s t, f)$. By assumption $f$ is non-zero. Suppose $s t=0$. Then $s^{-1} s t t^{-1}=0$. But $s^{-1} s \leq e=t t^{-1}$, so that $s^{-1} s t t^{-1}=s^{-1} s$. Hence $s^{-1} s=0$ which implies $s=0$, a contradiction. Thus $s t$ is also non-zero. Observe that

$$
(s t)^{-1}(s t)=t^{-1}\left(s^{-1} s\right) t \leq t^{-1} e t \leq t^{-1} t \leq f .
$$

Thus $(s t, f) \in C_{1}(S)$ and the product is well-defined.

Observe that $\exists(s, e) \cdot(t, f)$ precisely when $\mathrm{d}(s, e)=\mathbf{r}(t, f)$. It is now a simple matter to show that $\left(C_{1}(S), \cdot\right)$ is a category with identities $\{(e, e): e \in E(S) \backslash\{0\}\}$.

To show that $\left(C_{1}(S), \cdot\right)$ is right cancellative. Suppose that 


$$
(s, e) \cdot(t, f)=(u, i) \cdot(t, f)
$$

Then $(s t, f)=(u t, f)$ so that $s t=u t$. Thus $s t t^{-1}=u t t^{-1}$. But $e=t t^{-1}=i$ and $s^{-1} s, u^{-1} u \leq e$. Hence $s=u$ and $e=i$, and so $(s, e)=(u, i)$.

(ii) Observe first that the products $\left(s, s^{-1} s\right) \cdot\left(s^{-1}, s s^{-1}\right)$ and $\left(s^{-1}, s s^{-1}\right) \cdot\left(s, s^{-1} s\right)$ are defined, and that

$$
\left(s, s^{-1} s\right) \cdot\left(s^{-1}, s s^{-1}\right)=\left(s s^{-1}, s s^{-1}\right)=\mathbf{r}\left(s, s^{-1} s\right)
$$

and

$$
\left(s^{-1}, s s^{-1}\right) \cdot\left(s, s^{-1} s\right)=\left(s^{-1} s, s^{-1} s\right)=\mathrm{d}\left(s, s^{-1} s\right)
$$

Thus $\left(s, s^{-1} s\right)$ is invertible with inverse $\left(s^{-1}, s s^{-1}\right)$. Conversely, suppose that $(s, e)$ is an invertible element. Then there exists an element $(t, f)$ such that $(s, e) \cdot(t, f)$ and $(t, f) \cdot(s, e)$ are defined and

$$
(s, e) \cdot(t, f)=\mathbf{r}(s, e) \text { and }(t, f) \cdot(s, e)=\mathrm{d}(s, e) .
$$

Thus

$$
(s t, f)=\left(s s^{-1}, s s^{-1}\right) \text { and }(t s, e)=(e, e) .
$$

Then $e=t t^{-1}, f=s s^{-1}$, st $=s s^{-1}$ and $t s=e$. From $s t=s s^{-1}$ we obtain $s t s=s$. From $t s=e$ we obtain $t s t=e t=t t^{-1} t=t$. Thus $t=s^{-1}$. Hence $e=s^{-1} s$.

(iii) Let $(s, e)$ and $(t, e)$ be a pair of elements of $C_{1}(S)$ with a common domain. We suppose additionally that there are elements $\left(u, s s^{-1}\right)$ and $\left(v, t t^{-1}\right)$ such that

$$
\left(u, s s^{-1}\right)(s, e)=\left(v, t t^{-1}\right)(t, e) .
$$

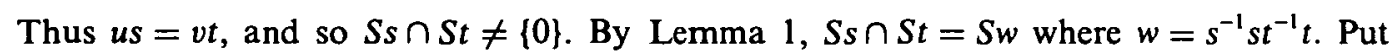
$a=t^{-1} t s^{-1}$ and $b=s^{-1} s t^{-1}$. Then $w=a s=b t$, and

$$
a^{-1} a \leq s s^{-1}, b^{-1} b \leq t t^{-1} \text { and } a a^{-1}=b b^{-1} \text {. }
$$

Thus $\left(a, s s^{-1}\right)$ and $\left(b, t t^{-1}\right)$ are well-defined elements of $C_{1}(S)$ and $\mathbf{r}\left(a, s s^{-1}\right)=\mathbf{r}\left(b, t t^{-1}\right)$. Also

$$
\left(a, s s^{-1}\right)(s, e)=\left(b, t t^{-1}\right)(t, f) .
$$

We shall show that the pair $\left(a, s s^{-1}\right),\left(b, t t^{-1}\right)$ is a pushout of the pair $(s, e),(t, f)$. Suppose that

$$
\left(x, s s^{-1}\right)(s, e)=\left(y, t t^{-1}\right)(t, f)
$$


Then $x s=y t=c w$ for some $c \in S$ such that $c^{-1} c \leq a a^{-1}=b b^{-1}$. But then $x s=c w=c a s$ and so $x s s^{-1}=c a s s^{-1}$. Hence $x=c a$. Similarly, $y=c b$. The pair $\left(c, a a^{-1}\right)$ is a well-defined element of $C_{1}(S)$ and

$$
\left(x, s s^{-1}\right)=\left(c, a a^{-1}\right)\left(a, s s^{-1}\right) \text { and }\left(y, t t^{-1}\right)=\left(c, a a^{-1}\right)\left(b, t t^{-1}\right) .
$$

The element $\left(c, a a^{-1}\right)$ is unique with these properties by right cancellation.

If $S=\{0\}$ then $C_{1}(S)$ is the empty category; this causes no problems.

The proof of the following is straightforward.

Corollary 3. Let $S$ be an inverse semigroup with zero, such that st $=0$ implies $s=0$ or $t=0$. Then $C_{1}(S)$ has pushouts of all pairs of elements with a common domain.

If $S$ is an inverse monoid with zero, then the element $(1,1)$ of $C_{1}(S)$ is a weak initial identity, since for every identity $(e, e)$ in $C_{1}(S)$, the pair $(e, 1)$ is an element of $C_{1}(S)$.

Theorem 4. For every inverse monoid with zero $S$, the category $C_{1}(S)$ is a $C R M$ category.

0-E-unitary inverse monoids can be characterised in terms of the properties of the category $C_{1}(S)$.

Theorem 5. Let $S$ be an inverse monoid with zero. Then $S$ is 0 -E-unitary if, and only if, $C_{1}(S)$ is a cancellative category.

Proof. Let $S$ be $0-E$-unitary and suppose that

$$
(a, e)(b, f)=(a, e)(c, f) .
$$

Then $a b=a c$. Thus $w=a^{-1} a b=a^{-1} a c$ and so $w \leq b, c$. Hence $w^{-1} w \leq b^{-1} c$. Suppose $w^{-1} w=0$. Then $a b=0$, a contradiction. Thus $w^{-1} w$ is a non-zero idempotent and so $b^{-1} c$ is an idempotent. But $b \mathcal{R} c$ and so $b^{-1} b \mathcal{R} b^{-1} c$. Thus $b^{-1} b=b^{-1} c$ and so $b=b b^{-1} c=c$. Hence $(b, f)=(c, f)$.

Conversely, suppose that $C_{1}(S)$ is left cancellative. We prove that $S$ is 0 -E-unitary. Let $e$ be a non-zero idempotent and suppose that $e \leq a$. Then

$$
\left(e, a a^{-1}\right)(a, 1)=\left(e, a a^{-1}\right)\left(a a^{-1}, 1\right)
$$

holds in $C_{1}(S)$. Thus by left cancellativity $(a, 1)=\left(a a^{-1}, 1\right)$ and so $a$ is an idempotent.

Having shown how to construct CRM-categories from inverse monoids, we now show how to construct inverse monoids from CRM-categories.

Let $C$ be a CRM-category and let 1 be the weak initial object. Let $C^{\prime}=\{x \in C: \mathrm{d}(x)=1\}$. 
If $x \in C^{\prime}$ then $C \cdot x=\{a \cdot x: a \in C\}$ is a subset of $C^{\prime}$. If $x, y \in C^{\prime}$ then the intersection $C \cdot x \cap C \cdot y$ is non-empty precisely when $x$ and $y$ can be completed to a commutative square; if $(s, t)$ is a pushout of $(x, y)$ then $s \cdot x=t \cdot y=z$, say. We define $(y * x),(x * y) \in C$ by

$$
z=(y * x) \cdot x=(x * y) \cdot y
$$

we also have that $C \cdot x \cap C \cdot y=C \cdot z$.

Let $x$ and $y$ be a pair of elements such that $\mathbf{r}(x)=\mathbf{r}(y)$. Then if $u \in C$ such that $\exists u \cdot x$ (and so $\exists u \cdot y$ ) we write $(u \cdot x, u \cdot y)=u \cdot(x, y)$. On the set of ordered pairs $(x, y)$ in $C^{\prime} \times C^{\prime}$ satisfying $\mathbf{r}(x)=\mathbf{r}(y)$ define a relation $\sim$ by

$$
(x, y) \sim\left(x^{\prime}, y^{\prime}\right) \Leftrightarrow(x, y)=u \cdot\left(x^{\prime}, y^{\prime}\right)
$$

for some invertible element $u \in C$. Then $\sim$ is an equivalence relation. Denote by $[x, y]$ the $\sim$-equivalence class containing the pair $(x, y)$. Let $J(C)$ denote the set of all $\sim$-equivalence classes with a zero adjoined; this latter point is another area of difference with Leech's construction.

Theorem 6. Let C be a CRM-category. Define a product on $J(C)$ as follows:

$$
[x, y] \otimes[w, z]=\left\{\begin{array}{l}
{[(w * y) \cdot x,(y * w) \cdot z] \quad \text { if } C \cdot y \cap C \cdot w \neq \emptyset} \\
0 \text { else }
\end{array}\right.
$$

and all other products equal to 0. Then $J(C)$ is an inverse monoid with zero.

In the semigroup $J(C)$ we have that

$$
[x, y]^{-1}=[y, x],[x, y]^{-1} \otimes[x, y]=[y, y], \text { and }[x, y] \otimes[x, y]^{-1}=[x, x]
$$

the idempotents are all the elements of the form $[x, x]$ for some $x \in C$ and the natural partial order is given by

$$
[x, y] \leq[w, z] \Leftrightarrow(x, y)=u \cdot(w, z) \text { for some } u \in C .
$$

The proof of the above theorem follows from my general theory of [17].

Put $J^{*}(C)=J(C) \backslash\{0\}$. The proof of the following is straightforward.

Theorem 7. Let $C$ be a CRM-category in which any two elements with a common domain have a pushout. Then the product of any two non-zero elements of $J(C)$ is nonzero. Consequently, $J^{*}(C)$ is an inverse semigroup without zero.

We now spell out the exact relationship between inverse monoids and CRMcategories. 
Theorem 8. Let $S$ be an inverse monoid with zero. Then $S$ is isomorphic to $J\left(C_{1}(S)\right)$.

The above theorem follows from [17] or it can be proved directly by modifying Leech's proof [20]. The isomorphism from $S$ to $J\left(C_{1}(S)\right)$ is given by $l(s)=\left[\left(s s^{-1}, 1\right),(s, 1)\right]$ and $\iota(0)=0$.

We shall now formulate necessary and sufficient conditions for the existence of a suitable prehomomorphism from a $0-E$-unitary inverse monoid $S$ to a primitive inverse semigroup. Observe first, that if $\theta: S \rightarrow T$ is a suitable prehomomorphism from an inverse monoid $S$ to a primitive inverse semigroup $T$, then the argument of Proposition 2.6(i) shows that the images of all non-zero elements of $S$ lie in a single group $\mathcal{H}$-class of $T$. Thus it is enough to consider the case where $T$ is a 0 -group.

Proposition 9. Let $S$ be a 0 -E-unitary inverse monoid with zero and let $\theta: S \rightarrow G^{0}$ be a 0-restricted idempotent pure prehomomorphism to a 0-group. Define $F: C_{1}(S) \rightarrow G$ by $F(s, e)=\theta(s)$. Then $F$ is a faithful functor.

Proof. The function $F$ is well-defined because $\theta$ is 0 -restricted and so if $s$ is nonzero then $\theta(s)$ is non-zero. To show that $F$ is a functor, observe that if $(e, e)$ is an identity in $C_{1}(S)$ then $\theta(e)$ is an idempotent in $G$ and so is equal to the identity of $G$. It remains to check that products are preserved. Suppose that $(s, e) \cdot(t, f)$ exists in $C_{1}(S)$. Then $s^{-1} s \leq e=t t^{-1}$. Thus by Proposition 2.1(iii), we have that $\theta(s t)=\theta(s) \theta(t)$. Hence $F((s, e)(t, f))=F(s t, f)$.

Finally, we show that $F$ is faithful. Let $(s, e),(t, e) \in C_{1}(S)$ such that $\mathrm{d}(s, e)=\mathrm{d}(t, e)$ and $\mathrm{r}(s, e)=\mathrm{r}(t, e)$ and $F(s, e)=F(t, e)$. The product $s^{-1} t$ is non-zero, otherwise $s s^{-1} t t^{-1}=s s^{-1}=0$, a contradiction. Thus $\theta\left(s^{-1} t\right)$ is a non-zero idempotent, and so, since $\theta$ is idempotent pure, $s^{-1} t$ is a non-zero idempotent. But $s \mathcal{R} t$ and so $s^{-1} s s^{-1} t$. Thus $s^{-1} s=s^{-1} t$ and $s=s s^{-1} t=t$. Hence $(s, e)=(t, e)$.

A necessary condition for the existence of a suitable prehomomorphism to a 0-group is that the corresponding CRM-category admit a faithful functor to a 0 -group. We now show that this condition is also sufficient.

Proposition 10. Let $C$ be a cancellative $C R M$-category and let $F: C \rightarrow G$ be $a$ faithful functor to a group $G$. Then there is suitable prehomomorphism $\theta: J(C) \rightarrow G^{0}$.

Proof. Define $\theta([x, y])=F(x)^{-1} F(y)$ and $\theta(0)=0$. We begin by checking that $\theta$ is a well-defined function.

Suppose that $[x, y]=\left[x^{\prime}, y^{\prime}\right]$. Then $(x, y)=u \cdot\left(x^{\prime}, y^{\prime}\right)$ for some invertible element $u$ in C. Thus

$$
\theta([x, y])=F(x)^{-1} F(y)=F\left(u \cdot x^{\prime}\right)^{-1} F\left(u \cdot y^{\prime}\right)=F\left(x^{\prime}\right)^{-1} F\left(y^{\prime}\right)=\theta\left(\left[x^{\prime}, y^{\prime}\right]\right) .
$$

Thus $\theta$ is a well-defined function. It is 0 -restricted by definition.

We now show that $\theta$ is a prehomomorphism by showing that it preserves the natural 
partial order and the restricted product. Suppose that $[x, y] \leq\left[x^{\prime}, y^{\prime}\right]$ in $J(C)$. Then by Theorem 6 , there exists $a \in C$ such that $(x, y)=a \cdot\left(x^{\prime}, y^{\prime}\right)$. But then

$$
\theta([x, y])=\theta\left[\left(a \cdot x^{\prime}, a \cdot y^{\prime}\right)\right]=F\left(a \cdot x^{\prime}\right)^{-1} F\left(a \cdot y^{\prime}\right)=F\left(x^{\prime}\right)^{-1}\left(y^{\prime}\right)=\theta\left(\left[x^{\prime}, y^{\prime}\right]\right) .
$$

To show that restricted products are preserved, suppose that $[x, y]\left[x^{\prime}, y^{\prime}\right]$ is a restricted product. Then $y=u \cdot x^{\prime}$ for some invertible element $u$ and so $[x, y]\left[x^{\prime}, y^{\prime}\right]=$ $\left[x, u \cdot y^{\prime}\right]$. Thus

$$
F\left([x, y]\left[x^{\prime}, y^{\prime}\right]\right)=F\left(\left[x, u \cdot y^{\prime}\right]\right)=\theta(x)^{-1} \theta(u) \theta\left(y^{\prime}\right)
$$

and

$$
F([x, y]) F\left(\left[x^{\prime}, y^{\prime}\right]\right)=\theta(x)^{-1} \theta(y) \theta\left(x^{\prime}\right)^{-1} \theta\left(y^{\prime}\right)=\theta(x)^{-1} \theta(u) \theta\left(y^{\prime}\right)
$$

It remains to show that $\theta$ is idempotent pure. Suppose that $\theta[x, y]$ is an idempotent. Then $F(x)^{-1}(y)=1$ and so $F(x)=F(y)$. But $[x, y] \in J(C)$ implies that $1=\mathbf{d}(x)=\mathbf{d}(y)$ and $\mathbf{r}(x)=\mathbf{r}(y)$. Thus $x$ and $y$ belong to the same hom-set. However $F$ is faithful, and so $x=y$. Hence $[x, y]$ is an idempotent.

Since $S$ is isomorphic to $J\left(C_{1}(S)\right.$ ) by Theorem 8 , any faithful functor from $C_{1}(S)$ to a group induces a suitable prehomomorphism from $S$ to a 0 -group. The construction of suitable prehomomorphism is summarised in the following theorem.

Theorem 11. Let $S$ be a 0 -E-unitary inverse monoid. Then $S$ admits a 0 -restricted idempotent pure prehomomorphism to a primitive inverse semigroup if, and only if, the category $C_{1}(S)$ admits a faithful functor to a group.

The appropriate generalisation of the fundamental group of a monoid is the concept of the "fundamental groupoid" of a category.

Theorem 12. For every category $C$ there exists a groupoid $U(C)$ and a functor $\eta: C \rightarrow U(C)$ such that $\eta(C)$ generates $U(C)$ and for every functor $\theta: C \rightarrow G$ to a groupoid $G$ such that $\theta(C)$ generates $G$ there exists a unique functor $\theta^{*}: U(C) \rightarrow G$ such that $\theta^{*} \eta=\theta$.

The fundamental groupoids of CRM-categories are rather special.

Lemma 13. Let $C$ be a CRM-category. Then $U(C)$ is a connected groupoid.

Proof. Let 1 be the weak initial identity of $C$. Then for every identity $e \in C$ there is an element $x \in \operatorname{hom}(1, e)$. Thus $\eta(x) \in \operatorname{hom}(\eta(1), \eta(e))$. But every identity in $U(C)$ is of the form $\eta(e)$ for some identity $e$ in $C$. Thus $U(C)$ is a connected groupoid. 
We now have the following

Theorem 14. (i) $A C R M$-category $C$ admits a faithful functor to a group if, and only if, the functor $\eta: C \rightarrow U(C)$ is faithful.

(ii) An inverse monoid $S$ admits a suitable prehomomorphism to a primitive inverse semigroup if, and only if, $\eta: C_{1}(S) \rightarrow U\left(C_{1}(S)\right)$ is faithful.

Proof. (i) Suppose that $\theta: C \rightarrow G$ is a faithful functor to a group. Without loss of generality, we may assume that $G$ is generated by the image of $\theta$. Thus by Theorem 12, there exists a unique functor $\theta^{*}: U(C) \rightarrow G$ such that $\theta=\theta^{*} \eta$. Now let $x, y \in \operatorname{hom}(e, f)$ where $\eta(x)=\eta(y)$. Then $\theta(x)=\theta(y)$. But $\theta$ is faithful. Thus $x=y$. Hence $\eta$ is faithful.

Conversely, suppose that $\eta: C \rightarrow U(C)$ is a faithful functor. By Lemma 13,U(C) is a connected groupoid. Let $G$ be any endomorphism group of $U(C)$. Then from [8] there is an equivalence of categories $\phi: U(C) \rightarrow G$. But $\phi$ is, in particular, faithful, and so $\phi \eta: C \rightarrow G$ is a faithful functor to a group.

(ii) By Theorem 11, $S$ admits a suitable prehomomorphism to a primitive inverse semigroup precisely when $C_{1}(S)$ admits a faithful functor to a group. The result now follows by (i).

If a 0 -E-unitary inverse monoid admits a suitable prehomomorphism then we would like to find a canonical such prehomomorphism. We shall use the following result from [8].

Theorem 15. Let $G$ be a groupoid. Then there is a group $\mathcal{G}(G)$ and a functor $\kappa: G \rightarrow \mathcal{G}(G)$ such that if $\theta: G \rightarrow H$ is any functor to a group then there exists a unique functor $\theta^{*}: \mathcal{G}(G) \rightarrow H$ such that $\theta^{*} \kappa=\theta$.

The proof of the following is straightforward.

Proposition 16. A category $C$ admits a faithful functor to a group if, and only if, the functor $\zeta=\kappa \eta: C \rightarrow \mathcal{G}(U(C))$ is faithful.

Let $S$ be a 0 -E-unitary inverse monoid which admits a suitable prehomomorphism to a 0-group. Then $\zeta: C_{1}(S) \rightarrow \mathcal{G}\left(U\left(C_{1}(S)\right)\right)=G$ is a faithful functor. By Proposition $10, \zeta$ induces a suitable prehomomorphism from $J\left(C_{1}(S)\right)$ to $G^{0}$. Composing this prehomomorphism with the isomorphism described after Theorem 8, we obtain a suitable prehomomorphism $\beta: S \rightarrow G^{0}$. It is characterised by the following property.

Theorem 17. Let $\theta: S \rightarrow T$ be any suitable prehomomorphism to a primitive inverse semigroup $T$ such that the image of $\theta$ generates $T$. Then there is a unique 0 -restricted prehomomorphism $\theta^{*}: G^{0} \rightarrow T$ such that $\theta^{*} \beta=\theta$.

Proof. We have already observed that $T$ must be a 0 -group, $H^{0}$ say. By Proposition 
9, $\theta$ induces a faithful functor $F: C_{1}(S) \rightarrow H$. The definition of $F$ implies that the image of $F$ generates $H$. Combining Theorem 12 and Theorem 15, there exists a unique functor $F^{*}: G \rightarrow H$ such that $F=F^{*} \zeta$. Define $\theta: G^{0} \rightarrow H^{0}$ to map 0 to 0 and to agree with $F^{*}$ on non-zero elements. Then $\theta^{*}$ is a well-defined (pre)homomorphism.

We now show that $\theta^{*} \beta=\theta$. By definition, $\beta(s)=\zeta\left(s s^{-1}, 1\right)^{-1} \zeta(s, 1)$ and $\beta(0)=0$. Thus for $s \neq 0$ we have that

$$
\theta^{*}(\beta(s))=F^{*}\left(\zeta\left(s s^{-1}, 1\right)^{-1}\right) F^{*}(\zeta(s, 1))=F\left(s s^{-1}, 1\right)^{-1} F(s, 1) .
$$

But by Proposition 9, we have that

$$
F\left(s s^{-1}, 1\right)^{-1} F(s, 1)=\theta\left(s s^{-1}\right) \theta(s) .
$$

However, $s s^{-1} \cdot s$ is a restricted product and so by Proposition 2.1 we have that $\theta\left(s s^{-1}\right) \theta(s)=\theta(s)$. Hence $\theta^{*} \beta=\theta$. It is easy to check that $\theta^{*}$ is unique with the stated properties.

Now that the general theory has been clarified, we can turn to applications. We begin by showing how the 0 -bisimple inverse monoid case can be recaptured from our general theory.

Proposition 18. Let $S$ be a 0-bisimple inverse monoid. Then the category $C_{1}(S)$ is equivalent to the monoid $R_{1}$.

Proof. Put $R=\operatorname{end}(1,1)$, the endomorphism monoid of $C_{1}(S)$ at the identity $(1,1)$. This monoid is isomorphic to $R_{1}$ when we define $\theta: R_{1} \rightarrow R$ by $\theta(a)=(a, 1)$. Let $(e, e)$ be any identity of $C_{1}(S)$. Because $S$ is 0 -bisimple and $e$ is non-zero there is an element $s \in S$ such that $e=s s^{-1}$ and $1=s^{-1} s$. The pair $\left(s, s^{-1} s\right)$ is an isomorphism in $C_{1}(S)$ satisfying $\mathrm{d}\left(s, s^{-1} s\right)=(1,1)$ and $\mathbf{r}\left(s, s^{-1} s\right)=(e, e)$. Thus $R$ is a full dense subcategory of $C_{1}(S)$ and so $R_{1}$ and $C_{1}(S)$ are equivalent categories.

Since equivalences are, in particular, faithful functors, it follows that $R_{1}$ admits a faithful functor to a group $G$ precisely when $C_{1}(S)$ admits a faithful functor to a group $G$. This is why the theory of suitable prehomomorphisms for 0-bisimple inverse monoids simplifies to the consideration of the monoid $R_{1}$.

Next we turn to the classical case of $E$-unitary inverse monoids. For this we need the category version of Theorem 2.12. The proof of the following may be found in [32].

Theorem 19. Let $C$ be a cancellative category satisfying Ore's condition: for all $x, y \in C$ with a common domain there exist elements $a, b \in C$ such that $a x=b y$. Then $C$ can be embedded in a groupoid; in particular, $U(C)=\eta(C)^{-1} \eta(C)$, and so is a groupoid of fractions of $C$. 
We may now recapture the classical theory of $E$-unitary inverse monoids.

Proposition 20. If $S$ is an E-unitary inverse monoid, then $U\left(C_{1}(S)\right)$ is a groupoid of fractions of $C_{1}(S)$. In particular, $C_{1}(S)$ can be embedded in a groupoid.

Proof. By Corollary 3, any two elements of $C_{1}(S)$ with a common domain have a pushout. Thus, in particular, $C_{1}(S)$ satisfies Ore's conditions of Theorem 19.

It follows by Theorem 14 that every $E$-unitary inverse monoid admits a suitable prehomomorphism to a group. We knew this already, of course. But by regarding $E$ unitary inverse monoids as being within the class of 0 - $E$-unitary inverse monoids, we have a new perspective on why this is true.

Von Karger proved the following generalisation of Theorem 2.14.

Theorem 21. Let $C$ be a cancellative category satisfying the mediation condition: for any $x, y \in C$ such that $a x=b y$ for some $a, b \in C$ then either $r x=y$ or $s y=x$ for some $r, s \in C$. Then $C$ can be embedded in a connected groupoid.

We shall now characterise those inverse monoids $S$ for which $C_{1}(S)$ satisfies the mediation condition of Theorem 21 .

Lemma 22. Let $S$ be an inverse monoid. Then $C_{1}(S)$ satisfies the mediation condition if, and only if, for all idempotents $e, f \in S$ if $e$ and $f$ have a non-zero lower bound then $e \leq f$ or $f \leq e$.

Proof. Suppose that $C_{1}(S)$ satisfies the mediation condition. Let $0 \neq i \leq e, f$. Then $(i, e),(e, 1),(i, f),(f, 1) \in C_{1}(S)$ are all well-defined and $(i, e)(e, 1)=(i, f)(f, 1)$. Thus by the mediation condition either $(e, 1)=(r, f)(f, 1)$ or $(f, 1)=(s, e)(e, 1)$ for some $(r, f),(s, e) \in C_{1}(S)$. Thus $e=r f$ or $f=s e$, and so $e f=e$ or $f e=f$. Hence $e \leq f$ or $f \leq e$.

Conversely, suppose that the idempotent condition holds. We show that $C_{1}(S)$ satisfies the mediation condition. Let $(x, i)(a, e)=(y, j)(b, e)$. Then $x a=y b$. Thus $(x a) a^{-1} a=(y b) b^{-1} b$ and so $(x a)^{-1}(x a) a^{-1} a=(x a)^{-1}(y b) b^{-1} b$. Put $i=(x a)^{-1}(x a) a^{-1} a$. If $i=0$ then $(x a) a^{-1} a=0$ which implies $x a=0$, a contradiction. Thus $i$ is a non-zero idempotent and $i \leq a^{-1} a, b^{-1} b$. Hence, $a^{-1} a \leq b^{-1} b$ or $b^{-1} b \leq a^{-1} a$. Suppose that the former holds. Then $a^{-1} a=a^{-1} a b^{-1} b$ and so $a=a b^{-1} b$. Consider the pair $\left(a b^{-1}, b b^{-1}\right)$. It is easy to check that this is a well-defined element of $C_{1}(S)$ and that $(a, e)=\left(a b^{-1}, b b^{-1}\right)(b, e)$. It is now evident that the mediation condition holds.

Following Birget $[1,2]$ we shall say that an inverse semigroup is unambiguous except at zero if the following two conditions hold:

(UA1) If $S a \cap S b$ is non-zero then $S a \subseteq S b$ or $S b \subseteq S a$.

(UA2) If $a S \cap b S$ is non-zero then $a S \subseteq b S$ or $b S \subseteq a S$. 
Because of the involution in an inverse semigroup it is easy to see that the above two conditions are equivalent.

Lemma 23. An inverse monoid is unambiguous except at zero if, and only if, for all idempotents $e, f \in S$ if $e$ and $f$ have a non-zero lower bound then $e \leq f$ or $f \leq e$.

Proof. Suppose that $S$ is unambiguous except at zero. Let $i \leq e, f$ be a non-zero lower bound of $e$ and $f$. Then $i \in S e \cap S f$. Thus, by assumption, Se $\subseteq S f$ or $S f \subseteq S e$. Hence $e \leq f$ or $f \leq e$.

Conversely, suppose that the idempotent condition holds and that $S a \cap S b$ is nonzero. Then $s a=t b \neq 0$ for some $s, t \in S$. Hence

$$
i=(s a)^{-1}(s a) a^{-1} a=(s a)^{-1}(t b) b^{-1} b
$$

is a non-zero idempotent. Thus $i \leq a^{-1} a, b^{-1} b$. Hence $a^{-1} a \leq b^{-1} b$ or $b^{-1} b \leq a^{-1} a$. Consequently, $S a \subseteq S b$ or $S b \subseteq S a$.

Combining the above two lemmas we have therefore proved the following.

Theorem 24. Let $S$ be an inverse monoid with zero. Then $S$ is unambiguous except at zero if, and only if, $C_{1}(S)$ satisfies the mediation condition.

The polycyclic monoids are unambiguous except at zero. By Theorems 24, 21 and 14 we have proved the following.

Theorem 25. Every 0-E-unitary inverse monoid which is unambiguous except at zero admits a suitable prehomomorphism to a primitive inverse semigroup.

\section{REFERENCES}

1. J.-C. Brrget, Iteration of expansions - unambiguous semigroups, J. Pure Appl. Algebra 34 (1984), 1-55.

2. J.-C. Birget, Arbitrary vs. regular semigroups, J. Pure Appl. Algebra 34 (1984), 57-115.

3. S. Bulman-Fleming, J. Fountain and V. Gould, Strongly $E^{*}$-unitary inverse semigroups, preprint University of York, England, July 1997.

4. A. H. Clifford, A class of d-simple semigroups, Amer. J. Math. 15 (1953), 541-556.

5. A. H. Clifford and G. Preston, The algebraic theory of semigroups, Volume 2 (Mathematical Surveys of the American Mathematical Society, no. 7, 1967, Providence, R.I.).

6. P. CoHn, Universal algebra (D. Reidel, 1981).

7. G. M. S. Gomes and J. M. HowIE, A P-theorem for inverse semigroups with zero, Portugal. Math. 53 (1996), 257-278.

8. Ph. Higgins, Notes on categories and groupoids (Van Nostrand Reinhold Company, London, 1971). 
9. B. VON KARGER, Embedding sequential calculus in relational calculus, preprint March 1994.

10. J. Kellendonk, Non-commutative geometry of tilings and gap labelling, Rev. Math. Phys. 7 (1995), 1133-1180.

11. J. Kellendonk, The local structure of tilings and their integer group of coinvariants, Comm. Math. Phys. 187 (1997), 115-157.

12. J. Kellendonk, Topological equivalence of tilings, J. Math. Phys. 38 (1997), 1823-1842.

13. M. V. LAwSON, The geometric theory of inverse semigroups III, unpublished preprint, University of Wales, Bangor, 1991.

14. M. V. LAwSON, The geometric theory of inverse semigroups I: E-unitary inverse semigroups, J. Pure Appl. Algebra 67(2) (1990), 151-177.

15. M. V. Lawson, The geometric theory of inverse semigroups II: E-unitary covers of inverse semigroups, J. Pure Appl. Algebra 83 (1992), 121-139.

16. M. V. LAwson, A class of actions of inverse semigroups, J. Algebra 179 (1996), 570-598.

17. M. V. Lawson, Constructing inverse semigroups from category actions, J. Pure Appl. Algebra, to appear.

18. M. V. LAwSON, The structure of 0-E-unitary inverse semigroups II: the general case, in preparation.

19. M. V. Lawson, The structure of 0-E-unitary inverse semigroups III: 0-restricted idempotent pure prehomomorphisms, in preparation.

20. J. LeECH, Constructing inverse monoids from small categories, Semigroup Forum 36 (1987), 89-116.

21. S. MACLANE, Categories for the working mathematician (Springer-Verlag, 1971).

22. S. W. Margolis and J. E. PIN, Inverse semigroups and extensions of groups by semilattices, J. Algebra 110 (1987), 277-297.

23. D. B. MCAlister, 0-bisimple inverse semigroups, Proc. London Math. Soc. (3) 28 (1974), 193-221.

24. D. B. MCAlister, ^-prehomomorphisms on inverse semigroups, Pacific J. Math. 67 (1976), 215-231.

25. D. B. MCAlister, Regular semigroups, fundamental semigroups and groups, $J$. Austral. Math. Soc. (Ser. A) 29 (1980), 475-503.

26. J. Meakin and M. SAPIR, Congruences on free monoids and submonoids of polycyclic monoids, J. Austral. Math. Soc. (Ser. A) 54 (1993), 236-253.

27. W. D. MUNN, Brandt congruences on inverse semigroups, Proc. London Math. Soc. (3) 14 (1964), 154-164.

28. M. Nivat and J.-F. Perrot, Une généralisation du monoide bicyclique, $C$. $R$. Acad. Sci. Paris A 271 (1970), 824-827.

29. M. Petrich, Inverse semigroups (John Wiley, 1984).

30. N. R. Reilly, Bisimple inverse semigroups, Trans. Amer. Math. Soc. 132 (1968), 101-114. 
31. M. B. SzendReI, A generalisation of McAlister's P-theorem for E-unitary regular semigroups, Acta Sci. Math. (Szeged) 51 (1987), 229-249.

32. C. A. WeIBEL, An introduction to homological algebra (Cambridge University Press, 1995).

\section{YsGol Fathemateg}

Prifysgol CyMru, Bangor

STRYD Y DEON

BANGOR

GWYNEDD LL57 1UT

WALES 